\title{
PRENDRE LA CRAIE. LA MOBILISATION DES ENSEIGNANTS REBELLES DANS LE NORD DE LA CÔTE D'IVOIRE (2002-2011)
}

Camille Popineau

\author{
Editions Karthala | « Politique africaine »
}

$2017 / 4 n^{\circ} 148$ | pages 27 à 48

ISSN 0244-7827

ISBN 9782811119655

Article disponible en ligne à l'adresse :

https://www.cairn.info/revue-politique-africaine-2017-4-page-27.htm

\section{Pour citer cet article :}

Camille Popineau, « Prendre la craie. La mobilisation des enseignants rebelles dans le Nord de la Côte d'Ivoire (2002-2011) », Politique africaine 2017/4 (n²148), p. 27-48.

DOI 10.3917/polaf.148.0027

Distribution électronique Cairn.info pour Editions Karthala.

(C) Editions Karthala. Tous droits réservés pour tous pays.

La reproduction ou représentation de cet article, notamment par photocopie, n'est autorisée que dans les limites des conditions générales d'utilisation du site ou, le cas échéant, des conditions générales de la licence souscrite par votre établissement. Toute autre reproduction ou représentation, en tout ou partie, sous quelque forme et de quelque manière que ce soit, est interdite sauf accord préalable et écrit de l'éditeur, en dehors des cas prévus par la législation en vigueur en France. Il est précisé que son stockage dans une base de données est également interdit. 


\section{LE DOSSIER}

CAMILle POPINEAU

\section{PRENDRE LA CRAIE. LA MOBILISATION DES ENSEIGNANTS REBELLES DANS LE NORD DE LA CÔTE D'IVOIRE (2002-2011)}

Cet article s'intéresse aux enseignants volontaires dans la zone rebelle de Côte d'lvoire entre 2002 et 2011. II montre que les relations de patronage au sein de la rébellion, la structuration du secteur scolaire et la présence d'un imaginaire où le syndicalisme étudiant est un vecteur d'ascension sociale ont permis la mobilisation d'acteurs dotés en capital scolaire. Ceux-ci sont investis dans des syndicats étudiants en zone rebelle afin d'intégrer l'administration rebelle, puis l'État. À la faveur de la guerre, l'engagement militant et la violence apparaissent comme des ressources pour se positionner dans les arcanes de l'État ivoirien. Ces trajectoires sont le signe de la reproduction en période de guerre de certaines dynamiques classiques d'ascension sociale ainsi que des modes d'exercice du pouvoir, interrogeant par là même les effets du conflit ivoirien sur les rapports de pouvoir au sein de l'État.

Le 19 septembre 2002, une tentative de coup d'État menée par des militaires se transforme en rébellion et mène à la scission du pays ${ }^{1}$. Le Nord est contrôlé par les Forces nouvelles² (FN) et le Sud par le régime de Laurent Gbagbo, et ce jusqu'en avril 2011. Une partie des habitants fuit la zone rebelle, mais beaucoup restent dans cette région «Centre-Nord-Ouest $(\mathrm{CNO})$ » couvrant $60 \%$ du territoire, faute d'alternative ou pour soutenir le mouvement rebelle. La rébellion limita essentiellement ses revendications à la fin des discriminations envers les «Nordistes», accusés d'être non-Ivoiriens depuis la seconde moitié des années $1990^{3}$.

\footnotetext{
1. Je tiens à remercier les évaluateurs de la revue, ainsi que les coordinateurs de ce numéro pour leurs questions stimulantes, leurs demandes de précisions et leurs remarques. Je remercie également Didier Péclard et Justin Pearce pour leurs remarques et suggestions à propos de ce travail.

2. Forces nouvelles est le nom donné à la coalition de trois groupes rebelles: le Mouvement patriotique de Côte d'Ivoire (MPCI), actifs au Centre et au Nord, le Mouvement populaire ivoirien du Grand Ouest (MPIGO) et le Mouvement pour la justice et la paix (MJP), actifs dans l'Ouest. La coalition se crée en 2003.

3. Voir, notamment, F. Akindès (dir.), Côte d'Ivoire: la réinvention de soi dans la violence, Dakar, Codesria, 2011; J.-P. Dozon, «La Côte d'Ivoire au péril de l'ivoirité. Genèse d'un coup d'État», Afrique contemporaine, $\mathrm{n}^{\circ} 193,2000$, p. 13-23.
} 
Suite à l'éclatement de la guerre, l'État rappela ses agents et l'ensemble des administrations fermèrent sur le territoire contrôlé par la rébellion. Mais le conflit perdurant, des services publics se reformèrent peu à peu, par le biais d'acteurs variés tels que des fonctionnaires restés dans la zone, des étudiants, des bénévoles, ainsi que des combattants rebelles. Parmi les secteurs ayant continué de fonctionner, on peut mentionner l'école, la santé, l'état civil, la communication ou encore les douanes, dont le degré de fonctionnement fut variable selon les régions et les périodes. Les FN n'ont pas cherché à investir immédiatement l'ensemble des champs d'intervention publique. Dans le domaine scolaire, c'est la mobilisation d'enseignants titulaires restés sur place ainsi que d'étudiants «volontaires ${ }^{4}$ » pour enseigner qui a permis la réouverture effective des salles de classe dès janvier $2003^{5}$. Tout au long de la guerre, ces enseignants de la zone CNO ont développé des liens forts avec les chefs rebelles, qui s'expliquent notamment par la proximité sociale de ces deux groupes. En effet, la formation du secteur scolaire est le fruit de l'alliance de deux générations d'étudiants, d'une part celle des leaders rebelles, anciens étudiants et leaders syndicaux à la fin des années 1990, et d'autre part celle des enseignants volontaires, encore étudiants au collège, au lycée ou à l'université en 2002 mais contraints d'arrêter leurs études à cause de l'éclatement de la crise.

La crise ivoirienne a donné lieu à de nombreuses publications, notamment sur son ancrage historique ${ }^{6}$, les registres identitaires mobilisés et les conceptions de la citoyenneté liées au conflit ${ }^{7}$, l'engagement des jeunes combattants ${ }^{8}$, les espaces de sociabilité abidjanais9, l'ancrage syndical des Jeunes patriotes

4. Ces étudiants se sont autoproclamés «enseignants volontaires», terme que nous reprendrons dans ce texte mais qu'il convient de distinguer du statut de «volontaire» introduit en Afrique de l'Ouest dans les années 1990. En effet, les «enseignants volontaires » interrogés n'enseignaient pas avant l'éclatement de la guerre et ne revendiquaient pas de filiation avec les volontaires engagés par l'État de Côte d'Ivoire au Sud.

5. À partir de cette date, les cours du primaire et du collège ont progressivement repris dans l'ensemble de la zone. De nombreux élèves ont fui et les effectifs étaient de ce fait largement réduits. 6. Voir, par exemple, F. Akindès (dir.), Côte d'Ivoire: la réinvention de soi..., op. cit. ; J.-P. Dozon, Les clefs de la crise ivoirienne, Paris, Karthala, 2011.

7. R. Marshall-Fratani, «The War of "Who Is Who" : Autochtony, Nationalism, and Citizenship in the Ivoirian Crisis?», African Studies Review, vol. 49, n² 2, 2006, p. 9-43.

8. Voir R. Banégas, «Côte d'Ivoire: les jeunes "se lèvent en hommes". Anticolonialisme et ultranationalisme chez les Jeunes patriotes d'Abidjan", Les études du Ceri, n 137, 2007, p. 1-53.

9. Voir A. Cutolo et R. Banégas, «Gouverner par la parole: parlements de rue, pratiques oratoires et subjectivation politique en Côte d'Ivoire», Politique africaine, $\mathrm{n}^{\circ} 127,2012$, p. 21-48. Sur les espaces de sociabilité des Nordistes, voir S. Vincourt et S. Kouyaté, «Ce que "parler au grin" veut dire: sociabilité urbaine, politique de la rue et reproduction sociale en Côte d'Ivoire", Politique africaine, $\mathrm{n}^{\circ} 127,2012$, p. 91-108. 
pro-Gbagbo $^{10}$, ou encore le lien entre patriotisme, violence et religion ${ }^{11}$. Cependant, en comparaison de la production académique consacrée à la zone gouvernementale, on constate une absence relative de recherches sur le Nord pendant la guerre ${ }^{12}$. Le fonctionnement de ce territoire, ses acteurs et ses évolutions constituent pourtant un cas particulièrement intéressant pour comprendre la possibilité de mobilisation dans une zone rebelle et les transformations des modes d'exercice du pouvoir en période de conflit.

Cet article apporte un éclairage sur le fonctionnement interne de la rébellion et amorce ainsi une sociologie des FN, en prenant pour objet la mobilisation d'un groupe d'étudiants dans le secteur scolaire. La multiplication des «volontaires» pendant la guerre n'est pas propre à la zone rebelle: la zone gouvernementale a également ouvert des écoles relais pour faire face à l'arrivée des réfugiés ayant fui la rébellion. Les enseignements y étaient en partie dispensés par des volontaires appelés «vacataires», dont le recrutement répondait à peu près aux mêmes logiques ${ }^{13}$. Il s'agira cependant, dans cet article, d'étudier l'engagement syndical des volontaires du Nord qui, du fait de leur trajectoire, de leurs relations avec les leaders rebelles et de leur présence dans la zone rebelle, a largement contribué à l'intégration des enseignants volontaires au niveau national à la fin du conflit.

L'école est ici un site d'observation des relations de pouvoir ${ }^{14}$, de la formation de réseaux locaux et d'alliances au sein des FN. La plupart des étudiants qui sont restés dans la zone CNO après la division du pays en deux ont soit pris les armes, soit investi le domaine scolaire en devenant enseignants volontaires. Ils se sont constitués en tant que groupe social mobilisé à travers différentes associations dont les objectifs et les modes d'action rappellent ceux des syndicats des années 1990. L'étude du secteur scolaire en zone rebelle,

10. Voir Y. Konate, «Les enfants de la balle. De la Fesci aux mouvements de patriotes», Politique africaine, $\mathrm{n}^{\circ} 89,2003$, p. 49-70.

11. Voir M. Miran-Guyon, Guerres mystiques en Côte d'Ivoire: religion, patriotisme, violence (2002-2013), Paris, Karthala, 2015.

12. À l'exception toutefois des travaux de T. Förster, «Maintenant, on sait qui est qui: Statehood and Political Reconfiguration in Northern Côte d'Ivoire», Development and Change, vol. 41, $\mathrm{n}^{\circ} 4$, 2010, p. 699-722 ; «Dialogue Direct: Rebel Governance and Civil Order in Northern Côte d'Ivoire», in A. Arjona, N. Kasfir et Z. Mampilly (dir.), Rebel Governance in Civil War, New York, Cambridge University Press, 2015, p. 203-225; mais aussi de M. Fofana, «Les jeunes dans la rébellion du nord de la Côte d'Ivoire: les raisons de la mobilisation ", Afrika Focus, vol. 24, n 1, 2011, p. 51-70; et enfin de K. Heitz-Topka, «Power-Sharing in the Local Arena: Man - A Rebel-Held Town in Western Côte d'Ivoire», Africa Spectrum, vol. 44, n³, 2009, p. 109-131.

13. Par exemple, un niveau troisième était requis pour enseigner au primaire et un niveau terminale pour le secondaire. Voir M. Chelpi-Den Hamer, «How to Certify Learning in a Country Split into Two by a Civil War? Governmental and Non-Governmental Initiatives in Côte d'Ivoire, 2002-06», Research in Comparative and International Education, vol. 2, n 3, 2007, p. 191-209.

14. Sur ce sujet, voir le dossier de Politique africaine, «Faire l'école», ainsi que l'introduction d'Hélène Charton, Politique africaine, $\mathrm{n}^{\circ} 139,2015$. 
en particulier des trajectoires, des profils et des répertoires d'action des enseignants, donne à voir des stratégies collectives d'ascension sociale proches de celles qui ont existé dans les années 1990, voire sous la présidence de Houphouët-Boigny (1960-1993), durant lesquelles le syndicalisme joue un rôle primordial. Dans le cas ivoirien, la guerre civile et la violence participent à une forme de reproduction des structures sociales et des luttes démocratiques des années 1990, reformulées par la lutte patriotique dans la guerre. Ces dynamiques de reproduction passent par la formation d'une alliance, en zone rebelle, entre les étudiants du Nord devenus enseignants, les leaders civils rebelles - eux-mêmes pour la plupart anciens étudiants et syndicalistes -, et les commandants de zone, généralement d'anciens militaires exilés suite au coup d'État de 1999 et à la répression menée par Robert Gueï ${ }^{15}$.

La dimension de patronage générationnel entre anciens leaders syndicaux et chefs de guerre, d'une part, et étudiants, d'autre part, constitue un élément crucial dans le fonctionnement des Forces nouvelles. Ces alliances en zone rebelle et la configuration particulière de la rébellion débouchent sur la reproduction d'un modèle de mobilisation où, à la faveur de la guerre, des acteurs dotés en capital scolaire s'investissent dans des syndicats et réussissent à intégrer l'administration rebelle, puis l'État ivoirien. Cette hypothèse pourrait aider à mieux comprendre les spécificités de la sortie de crise en Côte d'Ivoire en ce qu'elle renseigne certaines logiques de reproduction des groupes sociaux, des répertoires d'action et des figures de la réussite dans un contexte de crise. Nous verrons ainsi que les étudiants du Nord, dont les discours sont teintés d'une éthique enseignante et syndicaliste, constituent une nouvelle génération politique ${ }^{16}$ qui a réussi à convertir son enrégimentement dans les instances éducatives de la rébellion en capital social et en position de «pouvoir». Plus largement, l'expérience des enseignants volontaires dans la zone rebelle a renforcé les modes de recrutement contractuel dans le secteur scolaire de Côte d'Ivoire dans la période post-crise.

15. Les branches armée et civile de la rébellion ont ainsi en commun une expérience de la répression, de la prison, et de l'exil politique à l'intérieur ou à l'extérieur du pays, notamment au Burkina Faso. 16. L'hypothèse d'une nouvelle génération politique issue des rangs des syndicats étudiants a déjà été posée, notamment par Richard Banégas dans son travail sur les Jeunes patriotes. Cet article permet de valider cette hypothèse en l'élargissant aux acteurs de la zone rebelle et en précisant les mécanismes par lesquelles cette dynamique est rendue possible. Voir notamment R. Banégas, «La politique du "gbonhi". Mobilisations patriotiques, violence milicienne et carrières militantes en Côte-d'Ivoire», Genèses, n 81, 2010, p. 25-44. 
Prolongeant une recherche antérieure ${ }^{17}$, cet article est construit sur la base d'un corpus de 50 entretiens menés à Bouaké, Abidjan et Korhogo aux printemps 2016 et 2017 auprès d'ex-enseignants volontaires de la zone rebelle, d'ex-rebelles, de fonctionnaires, d'étudiants, de syndicalistes et de civils ayant vécu dans la zone $\mathrm{CNO}$ pendant la crise, ainsi que sur des documents obtenus auprès d'enquêtés, d'associations, d'ONG et d'anciens directeurs d'école. Je mets d'abord en évidence que l'institution scolaire rebelle ne rompt pas avec les pratiques de l'État ivoirien, en retraçant la constitution du secteur éducatif dans la zone rebelle. Ensuite, je montre que ce contexte est propice à la formation d'un groupe d'enseignants volontaires dont la mobilisation syndicale reprend une trajectoire comparable à celle des mouvements étudiants des années 1990. Je conclus sur le double succès de cette mobilisation, d'abord grâce à l'intégration des leaders du mouvement enseignant dans l'administration rebelle à partir de 2006-2007, puis au recrutement de plusieurs milliers de volontaires dans la fonction publique ivoirienne à partir de 2010. Réinscrit dans une temporalité plus longue, ce processus d'intégration s'inscrit dans une continuité par rapport à la politique d'incorporation des élites syndicales par l'État ivoirien.

\section{LA CONSTITUTION D'UN SECTEUR ÉdUCATIF EN ZONE REBELLE}

La rébellion ivoirienne se distingue d'autres rébellions en ce qu'elle a cherché à calquer son gouvernement sur le fonctionnement de l'État et non à créer un nouvel ordre politique. La restructuration d'un certain nombre d'administrations pourvoyeuses de services et la création de médias (une chaîne de télévision, une radio, un journal) en sont des indices, tout comme le réemploi de certains modèles de «bonne gouvernance» dans le secteur scolaire au Nord ${ }^{18}$. Ainsi, le ralliement à la cause des rebelles ou l'origine nordiste n'étaient par exemple pas des conditions préalables ${ }^{19}$ pour enseigner dans la zone rebelle, ce qui explique la variété de statut des acteurs qui s'y sont investis, et notamment la présence concomitante de titulaires, d'enseignants du privé, de retraités ou encore de volontaires.

17. C. Popineau, Faire l'école, faire l'État. Dynamiques rebelles de reproduction d'un ordre étatique en Côte d'Ivoire (2002-2010), Mémoire de Master 2, Paris, Université Paris 1, 2016. Cette recherche se poursuit aujourd'hui dans le cadre d'un doctorat.

18. Ibid.

19. Un certain nombre de mes enquêtés étaient par exemple originaires du Sud de la Côte d'Ivoire. Le principal critère de sélection était en réalité le niveau d'étude. 


\section{La structuration progressive du secteur scolaire en zone rebelle}

$\mathrm{Au}$ lendemain de l'éclatement de la crise, les discussions, notamment au sein des grins ${ }^{20}$ de Bouaké, ont rapidement débouché sur des initiatives privées dans le domaine scolaire. Entre octobre et décembre 2002, la question scolaire a été progressivement prise en charge par des structures associatives et humanitaires locales qui ont mis en place un bureau et des représentants locaux dans les différentes régions contrôlées par la rébellion. Elles se chargeaient de comptabiliser les enfants et les enseignants restés dans la zone, et de sensibiliser les parents afin qu'ils envoient leurs enfants à l'école ${ }^{21}$. La recomposition du système scolaire s'est faite sur un modèle légaliste ${ }^{22}$ où l'organisation demeurait hiérarchique et bureaucratique, pensée essentiellement par les fonctionnaires restés sur place ${ }^{23}$ et les enseignants volontaires, avec l'appui de certains chefs rebelles ${ }^{24}$.

Dès janvier 2003, des écoles ont rouvert dans les quartiers de Bouaké encore habités ${ }^{25}$. Le Collectif des enseignants volontaires (Codev) de la zone rebelle fut fondé début 2003, mais ce fut finalement l'ONG «École pour tous » (EPT), fondée en janvier 2003 et gérée par des fonctionnaires, qui prit en main l'organisation scolaire de la zone. La forme «ONG» était stratégique, comme

20. Si les grins de thé existent dans toute l'Afrique de l'Ouest, ce sont en Côte d'Ivoire des espaces de sociabilité où les personnes originaires du Nord se réunissent et discutent au quotidien. Voir S. Vincourt et S. Kouyaté, "Ce que "parler au grin" veut dire...», art. cité.

21. Malgré leur importance historique significative dans le Nord, les établissements privés y ont rouvert très tardivement. On peut davantage parler d'une union des enseignants des secteurs privé et public au sein des mêmes écoles durant la guerre.

22. En ce sens que des structures légales et institutionnelles identiques à celles préexistantes ont ainsi été recréées, comme par exemple les Comités de gestion (Coges) qui gèrent en temps normal les finances des écoles.

23. Sur 11526 enseignants en service au Nord lors de l'éclatement de la crise, environ la moitié serait restée dans la zone rebelle. Voir É. Lanoue, «L'école à l'épreuve de la guerre. Vers une territorialisation des politiques d'éducation en Côte d'Ivoire?», Politique africaine, n 92, 2003, p. 130. La plupart de mes enquêtés m'ont expliqué ne pas avoir eu le choix du fait de la présence de leur famille sur place. D'autres sont partis puis revenus du fait du manque de travail dans le Sud, malgré les promesses d'emploi de Laurent Gbagbo. Si les fonctionnaires désavouaient l'usage de la violence par la rébellion, la majorité d'entre eux soutenait ses revendications politiques.

24. Les chefs rebelles distribuaient ponctuellement des sommes d'argent pour aider les civils à réorganiser certains services tels que l'éducation. C'est ce qu'explique notamment Mamadou Traoré, ancien membre d'École pour tous, lorsqu'il relate une entrevue avec Ibrahima Coulibaly dit «IB » à Ouagadougou, lors de laquelle il était venu lui demander une aide financière pour les écoles de Boundiali. Voir M. Traoré, La craie et la kalachnikov. Mon combat pour sauver l'école en zone CNO, Abidjan, Éditions Saint Sauveur, 2015, notamment p. 189-196.

25. Certains quartiers, notamment ceux situés dans la partie Sud de la ville et habités majoritairement par des Baoulé (groupe du centre de la Côte d'Ivoire dont Bouaké est la capitale), se sont particulièrement vidés lors de l'éclatement de la crise. 720 écoles ont rouvert leurs portes durant l'année scolaire 2002-2003. Voir M. Chelpi-Den Hamer, «How to Certify Learning... », art. cité. 
me l'expliquait un fonctionnaire retraité, car elle représentait une organisation non affiliée à la rébellion, permettant ainsi de collaborer avec des ONG internationales et des «partenaires du développement ${ }^{26}$ ».

En raison du manque d'enseignants titulaires, plusieurs milliers d'élèves et d'étudiants s'engagèrent en tant qu'enseignants volontaires. L'accroissement de la demande sociale concernant l'éducation, amplifiée par l'annulation des examens suite aux bombardements de Bouaké en novembre 200427, incita la branche politique des Forces nouvelles à investir davantage ce secteur, en créant un Comité de sauvetage de l'école, puis le Comité école examen (C2E), sorte de ministère de l'Éducation rebelle, dirigé par un militaire de formation. Son investissement dans le domaine scolaire est ainsi l'expression d'un processus de légitimation au niveau local, où les chefs rebelles ont cherché à transformer leur pouvoir militaire en une domination politique ${ }^{28}$. À partir de cette date, un système institutionnel a été mis en place, accompagné de solidarités entre fonctionnaires titulaires et étudiants devenus enseignants volontaires dans une relation d'aînés à cadets.

\section{Continuité des modèles}

La formation d'un secteur scolaire sur le modèle bureaucratique dans la zone rebelle apparaît comme une forme de renégociation par le bas de la place des Nordistes dans l'administration étatique, où l'école permet à la rébellion et aux acteurs investis en son sein de prouver leur capacité et leur légitimité à gouverner. D'autre part, l'État tient une place centrale dans les imaginaires de régulation de la société et, surtout, dans l'accès aux ressources et aux positions d'accumulation, notamment du fait de l'importance accordée au capital scolaire. L'engagement des étudiants dans l'enseignement en zone CNO s'inscrit en effet dans un modèle de réussite ou de «bonne carrière» présent dans l'imaginaire des jeunes Ivoiriens. Les trajectoires de réussite lors de la période coloniale passaient très souvent par les institutions étatiques valorisant le passage par l'institution scolaire. Ces canaux classiques d'ascension sociale se sont perpétués dans la période postcoloniale, où la figure de l'intellectuel diplômé qui acquiert une position de pouvoir dans

\footnotetext{
26. Entretien, Bouaké, 2 mars 2016.

27. Le 6 novembre 2004, l'armée ivoirienne bombarde un camp français de la Force Licorne à Bouaké, tuant 9 soldats français et un Américain, et faisant 38 blessés.

28. Le besoin de légitimation sociale et politique est un élément central de compréhension du fonctionnement des rébellions. À ce sujet, voir K. Schlichte, In the Shadow of Violence: The Politics of Armed Groups, Francfort, Campus Verlag, 2009, ainsi que A. Arjona et al. (dir.), Rebel Governance..., op. cit.
} 
l'administration d'État grâce à son capital scolaire est restée valorisée. Au-delà du titre, l'école et l'enseignement sont aussi considérés comme des accélérateurs de carrières et des tremplins pour obtenir des postes politiques. Si les restrictions budgétaires et la crise des années 1980 ont affaibli ce modèle de promotion sociale, l'engagement dans l'école rebelle d'étudiants nordistes espérant leur intégration dans la fonction publique une fois la guerre passée témoigne de la persistance d'un imaginaire de la réussite lié à l'administration et à l'école. Ces trajectoires incitent à nuancer l'idée de la disparition des «vieux modèles» de réussite sociale et économique dans la zone rebelle ${ }^{29}$. L'État apparaît toujours comme une ressource essentielle pour les jeunes qui sont nombreux à espérer devenir fonctionnaires, en continuité symbolique avec le rôle historique qu'ont joué les enseignants dans l'Afrique postcoloniale francophone ${ }^{30}$, particulièrement en Côte $\mathrm{d}^{\prime}$ Ivoire.

Ainsi, la figure de l'enseignant volontaire reprend un registre classique de la revendication de respectabilité bien illustré par la trajectoire de l'un d'entre eux, Dao Issa. Né à Boundiali, dans le Nord, il a été au collège à Man, puis au lycée à Korhogo. Ses parents sont d'origine modeste, son père briquetier, sa mère ménagère. Il s'est engagé très tôt dans les mouvements et la représentation étudiante: président de coopérative quand il était en primaire, président du conseil scolaire au collège, puis au lycée, avant de rejoindre des associations d'étudiants à l'université, où il a obtenu sa maîtrise en droit en 2001. Il devient membre du Front populaire ivoirien (FPI) en 1990 avec l'arrivée du multipartisme, alors qu'il n'est qu'au collège, puis passe au Rassemblement des républicains (RDR) à sa création en 199431, où il est président du comité de base là où il enseigne. Avec l'éclatement de la crise, il se porte volontaire pour devenir enseignant dans les écoles de la zone rebelle. Il devient membre du Mouvement des enseignants volontaires de Côte d'Ivoire (Mevci), le principal syndicat des enseignants volontaires de la zone rebelle, créé en janvier 2004. Il intègre la fonction publique en mars 2010 et, suite à une dissension avec le $\mathrm{Mevci}^{32}$, il crée la Coordination nationale des enseignants volontaires de

29. Cela interroge la part de créativité et de reproduction des nouvelles figures de la réussite souvent évoquées, en montrant que celles-ci sont souvent encore en lien étroit avec certains imaginaires d'accumulation plus anciens. Voir, à ce sujet, le dossier de Politique africaine, «Figures de la réussite et imaginaires politiques", 2001, $\mathrm{n}^{\circ} 82$.

30. Voir J.-H. Jézéquel, Les "mangeurs de craie". Socio-histoire d'une catégorie lettrée à l'époque coloniale. Les instituteurs diplômés de l'École normale William Ponty (c. 1900-c. 1960), Thèse de doctorat, Paris, EHESS, 2002.

31. On retrouve ce parcours (avec le passage d'un engagement dans le FPI au RDR en 1994) chez de nombreux enseignants volontaires, estimant que le RDR les représentait mieux. En effet, la création du RDR fait suite au vote de la loi de septembre 1994 sur l'ivoirité exigeant que tout candidat à l'élection présidentielle soit né de parents ivoiriens, eux-mêmes nés de parents ivoiriens

32. Alassane Ouattara avait en effet annoncé fin 2011, alors que le règlement de la situation des enseignants volontaires avait été intégré dans le programme présidentiel de ce dernier, que la date 
Côte d'Ivoire (Cneev-ci) en août 2011 dont il devient président ${ }^{33}$. On voit bien, à travers l'ascension de Dao Issa, que l'engagement syndical et les capitaux scolaires apparaissent comme essentiels. La volonté des enseignants d'intégrer l'État amène à penser que leurs représentations des trajectoires d'accumulation économique et symbolique se font, même en période de guerre, dans son sillage.

\section{L'État et la «bonne gouvernance» comme référents}

L'attachement des enseignants à l'État se retrouve également dans leurs représentations de l'autorité régulatrice. Si l'autorité politique était exercée par les FN, les enseignants volontaires ont constamment eu comme perspective la réconciliation et l'État est demeuré le référent dominant pendant la guerre. Lorsque je demandai au directeur d'EPT par qui les cours devaient être «reconnus », il désignait le pouvoir d'Abidjan, seul capable de faire passer des examens officiels et de valider légalement leurs activités sur le long terme ${ }^{34}$. Les enquêtés dépeignent l'autorité des FN comme transitoire et n'ayant pas vocation à perdurer. Cette idée transparaît dans le contenu des enseignements dispensés dans la zone rebelle, où les programmes scolaires officiels ont été suivis à la lettre. André Ouattara, ancien vice-président du C2E, m'expliquait que c'étaient «exactement les mêmes programmes qui étaient enseignés à Abidjan. Exactement. Sinon plus d'ailleurs! ${ }^{35} »$. Calquer le programme sur celui de la zone gouvernementale témoigne de l'importance dans les représentations d'inscrire le système éducatif du Nord dans la légalité, et ces propos laissent transparaître une volonté d'essayer de faire mieux que dans le Sud. Les acteurs cherchaient ainsi à se placer sous le patronage de l'État, en réclamant par exemple dès 2003 la réorganisation par celui-ci des examens nationaux dans les zones contrôlées par la rébellion ${ }^{36}$. Au-delà, l'enjeu était aussi d'améliorer l'administration scolaire par rapport au Sud. La gestion du

\footnotetext{
retenue pour leur entrée en fonction était le $1^{\text {er }}$ septembre 2011 au lieu de mars 2010, comme le revendiquaient ces derniers, estimant que la période la précédant avait fait office de stage. Leur salaire n'ayant toujours pas été versé à cette date, cela revenait à supprimer 18 mois de salaire. Dans ce débat, le Mevci estima que cette décision était juste tandis qu'une partie des volontaires s'éleva pour faire entendre leur voix, créant ainsi la Cneev-ci.

33. Notons ici que, contrairement au Mevci, la Cneev-ci était une coordination à visée nationale.

34. Entretien, Bouaké, 18 février 2016.

35. Entretien, Bouaké, 25 février 2016.

36. Malgré la baisse du taux de scolarisation dans la zone rebelle, les examens ont eu lieu avec un an de décalage dans la zone rebelle, sauf pour l'année 2004-2005 du fait des tensions suite au bombardement de Bouaké. À partir de 2006-2007, les examens étaient de nouveau organisés en même temps au Nord et au Sud.
} 
problème de la négociation des notes dans les salles de classe en est un bon exemple. Un enseignant volontaire me racontait ainsi :

«Et puis éviter aussi que les rebelles viennent avec les fusils à l'école, viennent intimider quelqu'un, parce que y avait tout ça aussi. [...] Souvent ils venaient à l'école avec les tenues, avec les fusils à l'école. Ils viennent comme ça parce que soit ils ont leur petite copine qui est à l'école, ils viennent en tenue, bon, si... si elle a une mauvaise note y a d'autres qui peuvent venir voir l'enseignant, tout ça, pour influencer ${ }^{37}$ !

À cause des tentatives quotidiennes des combattants pour négocier les notes de leurs petites amies, les directeurs d'école ainsi que certains chefs rebelles avaient interdit aux militaires d'entrer dans les écoles, et même de sécuriser celles-ci. Dans un contexte national où la négociation des notes est très courante en temps normal ${ }^{38}$ et où les bailleurs internationaux exercent une pression croissante sur les institutions scolaires pour éradiquer ces pratiques, les enseignants et les $\mathrm{FN}$ cherchaient ainsi à montrer un respect plus strict des règles par rapport au Sud afin d'apparaître comme une autorité faisant preuve d'exemplarité et, par-là, de légitimité à gouverner. En effet, l'organisation du secteur scolaire rebelle sur le modèle étatique relève de plusieurs facteurs. D'une part, on assiste à une configuration d'acteurs singulière où il y a à la fois une forte demande sociale d'éducation, et la présence d'enseignants titulaires, de fonctionnaires de l'éducation, de directeurs régionaux de l'éducation nationale à la retraite et la présence massive d'étudiants volontaires qui s'investissent dans l'école. Par ailleurs, les liens entre le Nord et le Sud n'ont pas été complètement rompus dans le domaine scolaire, comme l'atteste l'organisation de nombreuses missions de la part du ministère de l'Éducation nationale dans la zone rebelle ou encore l'organisation des examens nationaux dans le Nord. Ensuite, le mimétisme avec les pratiques étatiques peut s'expliquer par le sentiment de dévaluation du travail effectué par les enseignants dans la zone rebelle, les écoles du Nord étant considérées avec mépris par les gens du Sud et même rebaptisées «écoles maliennes ${ }^{39}$ ». La formation d'une école calquée sur les standards nationaux est ainsi à comprendre dans ce contexte. En laissant se déployer les efforts scolaires dans la zone, la rébellion a également œuvré à être mieux perçue par les populations locales, permettant ainsi aux chefs rebelles de se targuer d'avoir mis en

37. Entretien, Bouaké, 25 février 2016.

38. Sur ces pratiques, voir notamment É. Lanoue, «La société ivoirienne au fil des réformes scolaires: une politique d'éducation "intermédiaire" est-elle possible?», Autrepart, n 31, 2004, p. 93-108.

39. Pour une explication plus détaillée de ce point, voir M. Chelpi-Den Hamer, «How to Certify Learning...», art. cité. 
place un «bon gouvernement», quand bien même celui-ci était principalement le fait de la présence d'acteurs qui ne faisaient initialement pas partie de ses cadres.

\section{"PRENDRE LA CRAIE": LA FORMATION D'UN GROUPE SOCIAL POUR SOI}

Ce cadre scolaire définit les conditions rendant possible la formation d'un groupe social d'enseignants volontaire et la mobilisation de ces derniers pour la défense de leurs intérêts à court et à long terme.

\section{La formation d'un groupe social en zone rebelle}

Les enseignants de la zone rebelle étaient principalement divisés en deux groupes, celui des fonctionnaires et celui des enseignants volontaires. Cette division était d'abord d'ordre économique: alors que les fonctionnaires en place dans l'administration scolaire ont continué à percevoir leur salaire durant toute la guerre ${ }^{40}$, les volontaires n'étaient payés que très ponctuellement en sacs de riz par le Programme alimentaire mondial (PAM), par certains commandants de zone ou par le biais des $\operatorname{Coges}^{41}$. Ensuite, la différence d'âge était notable puisque les enseignants volontaires, étudiants répartis dans des classes d'âge allant du collège à la maîtrise (entre 15 et 30 ans), étaient plus jeunes que les fonctionnaires. Au sein des établissements scolaires ayant rouvert leurs portes, on a assisté à la formation d'une hiérarchie dans laquelle les fonctionnaires dominaient. Ces derniers ont en effet mis en avant une « expertise » et des «compétences » que les volontaires n'avaient pas, se rendant essentiels pour apporter le crédit dont l'école de la zone rebelle avait besoin. Un ancien fonctionnaire m'expliquait ainsi :

«Je me considère comme une personne morale dont on avait besoin pour apporter du crédit à l'école. [...] Les programmes, les enseignements à dispenser, on n'enseigne pas n'importe quoi, à n'importe qui! Nous, nous maîtrisons les textes de l'administration scolaire. Les textes pédagogiques. Donc, nous avons eu des missions dans les écoles, pour suivre les uns et les autres ${ }^{42}$.»

40. Les banques étant fermées, les enseignants titulaires faisaient l'aller-retour en zone gouvernementale tous les deux ou trois mois afin de récupérer leur salaire. Les femmes d'enseignants s'y rendaient souvent pour leurs maris car elles étaient moins inquiétées lors des contrôles aux barrages.

41. Comités de gestion réunissant de l'argent auprès des parents d'élèves pour payer les enseignants. 42. Entretien, Bouaké, 2 mars 2016. 
Cette hiérarchie se retrouve de manière concrète dans la composition des administrations des écoles de la zone rebelle, où les postes de proviseurs, de censeurs, d'économes et d'éducateurs étaient tenus par des titulaires. Selon les chiffres du ministère de l'Éducation nationale pour l'année 2002-200343, les postes d'enseignants dans la zone $\mathrm{CNO}$ auraient été occupés essentiellement par des enseignants volontaires (4465 volontaires contre 1767 fonctionnaires) alors que les postes de direction et d'administration des écoles l'étaient majoritairement par des fonctionnaires (438 fonctionnaires contre 205 volontaires). Lorsque ce n'était pas le cas, c'était souvent en raison d'un manque de fonctionnaires, notamment dans les milieux ruraux. Enfin, cette division du travail apparaît dans l'organisation des enseignements eux-mêmes dans certaines classes, notamment à partir du redéploiement de l'administration scolaire, en 2004 puis $2006^{44}$. Un enseignant volontaire m'expliquait que les enseignements dans les classes étaient assurés par un titulaire et un volontaire, et que ce dernier avait un rôle d'assistant, notamment pour les corrections ou en cas d'absence du titulaire ${ }^{45}$. L'ossature binaire de l'enseignement entre les volontaires et les titulaires était ainsi une source d'inégalités qui a participé à unifier le groupe des enseignants volontaires de la zone rebelle autour d'intérêts communs.

\section{Mémoire syndicale et représentations de la lutte estudiantine}

La structuration des enseignants volontaires en mouvement est à replacer dans l'histoire syndicale et dans la mémoire des luttes estudiantines ivoiriennes. Laurence Proteau a montré l'importance de l'école comme lieu d'émergence des conflits socio-politiques en Côte d'Ivoire depuis les années 196046. Le syndicalisme a constitué une voie privilégiée d'ascension sociale et d'accès à la reconnaissance socio-professionnelle. Le premier président ivoirien, Félix Houphouët-Boigny, a joué un rôle décisif dans le syndicalisme

43. Ministère de l'Éducation nationale, Rapport de la mission d'évaluation des enseignements du 22 au 25 septembre 2003 à Bouaké, septembre 2003, Abidjan, Ministère de l'Éducation nationale, p. 4; M. Chelpi-Den Hamer, «How to Certify Learning....», art. cité.

44. Un premier redéploiement de l'administration scolaire a été amorcé en 2004, lorsque l'État a appelé les fonctionnaires de l'éducation (enseignants et membres des directions régionales de l'éducation nationale) à retourner dans la zone rebelle, en lançant notamment des primes de redéploiement. Une seconde vague de redéploiement a eu lieu en 2006.

45. Entretien, Bouaké, 12 mars 2016. Cette pratique n'était cependant pas généralisée, les fonctionnaires étant moins nombreux que les enseignants volontaires, malgré le premier redéploiement de 2004. Elle a été en revanche relativement répandue à partir du second redéploiement, c'est-à-dire à partir de 2006-2007.

46. L. Proteau, Passions scolaires en Côte d'Ivoire. École, État et société, Paris, Karthala, 2002. 
agricole avant d'accéder au pouvoir. De plus, après l'indépendance, les deux syndicats du Parti démocratique de Côte d'Ivoire (PDCI), l'Union générale des travailleurs de Côte d'Ivoire et le Mouvement des étudiants et élèves de Côte d'Ivoire (Meeci), ont successivement formé les futurs cadres de l'État et du parti unique ${ }^{47}$. Le syndicalisme constituait également la matrice de l'opposition au régime de parti unique d'Houphouët-Boigny. En effet, dans les années 1980, les enseignants forment le premier syndicat indépendant de l'Union générale des travailleurs de Côte d'Ivoire et du PDCI : le Syndicat africain de la recherche et de l'enseignement supérieur (Synares). Laurent Gbagbo, élu président en 2000, était enseignant et ancien cadre du Synares. Par la suite, les principaux protagonistes de la guerre étaient d'anciens patrons syndicaux de la Fédération estudiantine et scolaire de Côte d'Ivoire (Fesci), formée en 1990 par des étudiants dont les enseignants étaient également des cadres du Synares. En effet, dans les années 1990, la Fesci était très proche des rivaux du PDCI - le Front populaire ivoirien (FPI) et le Rassemblement des républicains (RDR) - et elle constituait un espace de socialisation à la contestation politique ${ }^{48}$.

Comme tout élève ou étudiant de Côte d'Ivoire, les enseignants volontaires connaissaient et ont côtoyé la Fesci durant leur scolarité, et ce d'autant plus lorsqu'ils ont étudié à l'université. S'ils n'en ont pas tous été membres, l'ensemble des étudiants avaient en mémoire l'histoire récente du syndicat et les luttes violentes qui ont accompagné la fin des années 1990, et plus particulièrement le début des années 2000 avec l'éclatement de la guerre des machettes ${ }^{49}$ et de la dissidence fesciste, à l'université d'Abidjan, mais aussi de Bouaké. Les succès répétés des mobilisations de la Fesci expliquent en partie la constitution des enseignants volontaires en syndicat à travers le Mevci dans la zone rebelle, la lutte syndicale étant réputée efficace.

47. L'intégration des élites syndicales du Meeci dans l'État peut s'illustrer par de nombreux exemples: Hamed Bakayoko (ministre de l'Intérieur de 2011 à 2017 et actuel ministre de la Défense, ancien du Meeci), Djédjé Mady (ancien leader du Meeci qui devint secrétaire général du PDCI), Jean-Jacques Béchio (ancien leader du Meeci qui devint conseiller politique d'Alassane D. Ouattara), mais aussi Aly Kéita, Laurent Akoun, Dagou Lignon, Gnaoulé Oupoh, qui ont été des hauts cadres du FPI. Voir Y. Konate, «Les enfants de la balle...», art. cité.

48. La Fesci a vite été l'alliée des partis d'opposition lors de l'avènement du multipartisme, d'autant que, comme le rappelle Yacouba Konate, «sur les quarante partis reconnus, une bonne trentaine est dirigée par des enseignants». Il rappelle également que les quatre partis les plus engagés ont tous été fondés par des enseignants: le Front populaire ivoirien de Laurent Gbagbo, le Parti ivoirien des travailleurs (PIT) de Francis Wodié (professeur de droit, ancien secrétaire général du Synares et ancien ministre de l'Enseignement supérieur et de la recherche scientifique), l'Union des sociauxdémocrates (USD) de Bernard Zadi et le Parti socialiste ivoirien (PSI) de Moriféré Bamba. Voir Y. Konate, «Les enfants de la balle...», art. cité, p. 52.

49. Cette expression fait référence à la lutte qui a eu lieu en juin 2000 entre la Fesci et la dissidence, respectivement menées par Charles Blé Goudé et Guillaume Soro, qui ont fait des dizaines de morts dans les campus universitaires, à Abidjan comme à Bouaké. 


\section{La création d'un mouvement estudiantin en zone rebelle}

Le poids des syndicats est manifeste dans le récit que me faisait un enseignant du blocage des directions régionales de l'éducation nationale (DREN) en 2010: «En tant que syndicalistes, on voit les opportunités. Ils ont pas pu faire les examens ${ }^{50}$.» De même, un enseignant volontaire, parlant de leurs représentants auprès des chefs rebelles, expliquait que «vu le poids que nous avions sur le terrain, ils ne pouvaient pas s'en défaire, ils étaient obligés de travailler avec nous ${ }^{51}$ ». Ils exprimaient ainsi l'idée que «pour exister » sur la scène politique, il fallait être syndiqué. Leur statut de dominés par rapport aux fonctionnaires et la mémoire de la lutte syndicale fesciste expliquent la formation d'un mouvement indépendant de l'ONG EPT et du C2E, considérées comme incapables de se faire leurs porte-voix. Ce récit d'un volontaire montre l'importance de la défense d'intérêts propres à leur statut vis-à-vis de l'autorité:

«L'ONG même, nous négligeait, en réalité. Parce qu'ils avaient une vision autre que la nôtre. Pour eux, si d'aventure il devait y avoir des rémunérations, des dédommagements, ou bien même si on devait enclencher le processus de recrutement dans la fonction publique, les choses passeraient par eux. Vous voyez un peu? Et les choses passant par eux, ils allaient faire ce qu'ils voulaient faire en réalité. [...] nous avions des personnes infiltrées qui nous donnaient déjà des idées par rapport aux ambitions de l'ONG, concernant notre dossier. Ils voulaient s'accaparer le dossier et puis se tailler la plus grosse part du gâteau. C'est comme ça que nous avons anticipé les choses et qu'on a préféré ne même pas travailler avec eux ${ }^{52}$.»

Lorsque l'on interroge les enseignants volontaires, tous expliquent avoir «pris la craie», un engagement dans l'école qui équivaut selon eux à une «prise d'arme», à une véritable lutte, à la fois pour la cause rebelle et pour la défense de leurs intérêts. Organisé de manière autonome à partir de 2004 à travers le Mevci ${ }^{53}$, le bureau était constitué d'étudiants à l'université de Bouaké avant la crise. Le Mevci défendait les intérêts des enseignants non titulaires: leurs revendications étaient d'ordre financier sur le court terme, mais visaient à terme la reconnaissance de leur action et leur intégration à la fonction publique ivoirienne. Le Mevci a ainsi organisé la lutte des enseignants volontaires durant la guerre, en menant des négociations avec le ministère

\footnotetext{
50. Entretien, Bouaké, 9 mars 2016.

51. Entretien, Bouaké, $1^{\mathrm{er}}$ mars 2016.

52. Entretien, Bouaké, 19 février 2016.

53. L'année 2004 donne également lieu à un changement de leadership à la tête de la rébellion, où Guillaume Soro s'impose face à Ibrahim Coulibaly. S'ensuivit une structuration accrue des institutions et un découpage administratif et militaire en dix zones, ainsi que l'intégration dans l'administration de nombreux proches de Soro, notamment d'anciens syndicalistes étudiants.
} 
de l'Éducation du gouvernement de Laurent Gbagbo, en produisant des documents ainsi qu'en recensant les volontaires afin de faciliter leur intégration future $^{54}$. Pour chaque année, dans chaque établissement de l'ensemble de la zone, la liste des enseignants était établie, incluant leur nom, âge, lieu de naissance, niveau d'étude, numéros de téléphone, et un matricule leur était attribué. De même, si des cartes d'enseignants des FN avaient été créées ${ }^{55}$, le Mevci a ensuite créé les siennes afin de rendre le mouvement visible et de souligner son importance dans l'organisation du secteur scolaire en zone rebelle.

Même si tous les enseignants volontaires n'ont pas été des membres actifs de la Fesci, l'existence et les modes d'action de ce syndicat étudiant sont évoqués par mes interlocuteurs. La Fesci est connue sur les campus universitaires pour être l'actrice principale de la formulation des revendications estudiantines et scolaires, et le Mevci a repris ses codes oratoires et ses modes d'action. Un groupe d'enseignants volontaires fut particulièrement représentatif de ce mimétisme. L'impératif de la lutte présent dans les discours fescistes des années 1990 se retrouve dans la vivacité de leurs discours:

\footnotetext{
«On attendait les affectations, ça ne venait pas, et on a encore bousculé [...] et je vous dis que à chaque fois qu'il y a eu des avancées concernant la disponibilité des listes, quelle que soit l'avancée que nous avons obtenue, nous l'avions obtenue dans la lutte. Mes camarades et moi. [...] nous avons été ceux qui ont réellement fait basculer les choses au niveau des enseignants volontaires, parce que tout ce qui se faisait, tout ce qui avait comme actions à mener sur le terrain, que ce soit des sit-in, que ce soit des marches, que ce soit des grèves, c'est nous qui occupions surtout cela, et je pense que ça nous a vraiment aidés ${ }^{56}$.»
}

Cet enseignant, ancien membre de la Fesci au lycée à Bouaké, raconte également, faisant référence aux années de lutte où le FPI et le RDR formaient un front commun d'opposition: "nous-mêmes qui sommes les élèves de Laurent Gbagbo, qui nous a toujours dit que "rien ne s'obtient, tout s'arrache", il était intéressant qu'on puisse vraiment se servir de ses conseils qu'il a toujours donnés à la jeunesse ${ }^{57}$ ». Ces discours laissent transparaître un imaginaire de la lutte où le syndicalisme et la violence sont les seuls moyens d'obtenir satisfaction ${ }^{58}$.

\footnotetext{
54. Il s'agissait de prouver par la production de documents que les volontaires avaient bien enseigné, combien de temps, et d'avoir une liste exacte de noms pouvant être potentiellement transmise au gouvernement en cas d'intégration.

55. Cette carte servait à faciliter les déplacements et à éviter qu'ils soient taxés par les combattants aux barrages de la ville lors de leurs trajets entre le domicile et l'école.

56. Entretien, Bouaké, 1er mars 2016.

57. Entretien, Bouaké, $1^{\mathrm{er}}$ mars 2016.

58. À propos de l'importance croissante des répertoires violents en Côte d'Ivoire, voir F. Akindès (dir.), Côte d'Ivoire: la réinvention de soi..., op. cit.
} 
L'importance de la distinction fesciste se voit par ailleurs dans la division entre les acteurs de l'éducation concernant les pratiques et les modes d'action à privilégier. Un fonctionnaire titulaire resté dans la zone Nord et membre d'EPT dit ainsi, en parlant des volontaires:

«On n'avait pas la même manière d'appréhender les choses. Eux, ils avaient les méthodes fescistes. [...] Puisque les trois quarts étaient des étudiants, donc la fédération estudiantine de Côte d'Ivoire [Fesci] a des méthodes, nous on s'accommodait très mal à cela. Cette méthode c'était la brutalité, alors que nous on s'accommodait au dialogue et à la conciliation. C'est pas qu'ils étaient proches de la Fesci mais ils avaient déjà ancré en eux ce comportement fesciste ${ }^{59}$.»

Cette citation illustre la prégnance de l'imaginaire fesciste chez les jeunes, mais aussi le fait que les plus âgés (le fonctionnaire en question ayant environ 20 ans de plus que les volontaires), ayant fait leurs études avant la naissance de la Fesci, ont conscience de la centralité de la Fesci parmi les étudiants ivoiriens. On constate une fracture générationnelle entre les aînés, déjà enseignants et fonctionnaires, et les cadets, étudiant dans un contexte socio-économique qui n'offre plus l'assurance d'un accès à la reconnaissance sociale depuis la réduction des budgets de la fonction publique suite à la mise en place des plans d'ajustement structurel limitant les recrutements dans les années 1990. L'«impératif syndicaliste» présent dans les discours est à comprendre dans ce rapport des étudiants à la Fesci et dans un contexte de difficultés sociales et professionnelles rencontrées à la sortie des études, où le syndicalisme constitue - peut-être plus qu'avant - une voie d'accès privilégiée aux postes de représentants politiques, mais aussi plus largement à un statut d'adulte et à une reconnaissance sociale. À cet égard, la présence de Guillaume Soro à la tête de la rébellion a largement participé à l'identification des jeunes nordistes à la lutte des insurgés et à leur implication dans les institutions reformées dans la zone CNO. En effet, la branche politique de la rébellion était essentiellement constituée d'anciens fescistes ayant fait partie de la dissidence menée par Guillaume Soro en 2000, s'opposant au secrétaire général en place, Charles Blé Goudé.

\section{LA RÉUSSITE de LA MOBILISATION ESTUdiANTINE}

L'homologie entre les luttes syndicales des années 1990 et celle des années 2000 dans le Nord se prolonge au-delà de la guerre. Les parcours des étudiants entre 2006 et aujourd'hui témoignent d'une ascension sociale sanctionnée par

59. Entretien, Bouaké, 25 février 2016. 
l'obtention du statut de fonctionnaire. Cette stratégie d'intégration des leaders syndicaux s'inscrit dans une continuité avec la politique d'incorporation des élites syndicales au sein de la fonction publique depuis la décolonisation.

\section{Logiques de cooptation dans l'administration rebelle}

À mesure que le secteur scolaire s'organisait, les FN ont coopté certains enseignants volontaires au sein de leur administration. Présidents, viceprésidents, secrétaires, les leaders des associations d'enseignants volontaires ont accédé à des positions importantes dans l'administration rebelle, en tant que représentants de la jeunesse ou des enseignants, ou encore en tant que conseillers techniques. Ces recrutements répondaient par ailleurs à des critères tels que le niveau d'étude et la motivation. On note ici la reproduction en période de guerre de certaines dynamiques de cooptation. Si la rébellion s'est d'abord déchargée de la question scolaire, la cooptation de leaders parmi les étudiants permit aux chefs rebelles de se réapproprier le processus scolaire et de s'y faire une place. Elle leur donna aussi l'occasion de disposer de relais au sein du personnel éducatif, et surtout de profiter de la capacité des étudiants à mobiliser. Un ancien volontaire me le racontait: "souvent quand y avait des grèves, des manifestations, souvent ils [les FN] demandaient l'appui des enseignants pour les soutenir, de sorte à galvaniser un peu ${ }^{60}$ ». Mais ce fut également un moyen d'anticiper la contestation sociale et d'étouffer dans l'œuf celle des volontaires eux-mêmes. Selon un enseignant:

«Il peut y avoir des plaintes, çà et là, pour dire «mais attend, ils sont en train de nous brimer là", et quand c'est comme ça, le bon leader, Soro Guillaume à l'époque, là, qu'est-ce que tu fais? Tu regardes dans la population toutes les couches de la société civile, tout ce qui s'occupe de l'éducation, de l'enseignement et tout, et tout là, ceux qui émergent comme les leaders là-bas, là, tu les mets dans ton cabinet, pour pouvoir avoir le retour plus facilement ${ }^{61}$.»

La cooptation s'est traduite par un encadrement des grèves des enseignants. Un collégien, devenu enseignant volontaire, le relate en ces termes: «On les empêchait pas mais on les encadrait dans leurs grèves. Bon "encadrer", c'est parce que généralement dans les grèves y a des casses, donc tout ça il fallait la sécurité pour qu'il n'y ait pas la violence ${ }^{62} »$. Un groupe potentiellement contestataire se forma ainsi, et sa force numérique et symbolique amena le

60. Entretien, Bouaké, 18 février 2016.

61. Entretien, Bouaké, 1 ${ }^{\mathrm{er}}$ mars 2016.

62. Entretien, Bouaké, 19 février 2016. 
pouvoir rebelle à les intégrer. De ce point de vue, la rébellion n'a pas rompu avec les modes classiques d'exercice du pouvoir de l'État ivoirien.

Une opposition s'est formée peu à peu au sein du Mevci, débouchant sur la création en 2010 du Cneev-ci. La dissidence semblait recouper des rapports de force au sein de la rébellion, notamment l'opposition entre les clans Soro et $\mathrm{IB}^{63}$, les premiers valorisant davantage une approche politique prônant la négociation diplomatique et les seconds rejetant cette méthode ${ }^{64}$. Cette opposition se retrouve en effet dans les discours des dissidents, pour qui la cooptation des leaders du Mevci au sein du secrétariat général des FN aurait ramolli le mouvement et l'aurait rendu inefficace car moins virulent. Un dissident me raconte leur lutte en 2011 pour la perception de leur salaire après leur intégration à la fonction publique:

«Nous, nous nous sommes démarqués à un certain moment parce que nous trouvions qu'il y avait une certaine lenteur, donc ça fait qu'on a créé cette dissidence-là, que nous avons pris nos responsabilités, que nous avons lutté jusqu'au bout, jusqu'à ce qu'on perçoive nos salaires! [...] Nous avons travaillé 32 mois sans être payés. Et au moment où on revendique pour cela, d'autres estiment qu'il n'est pas important de revendiquer [...] Alors qu'à ce moment-là, on a des camarades qui sont morts [de faim]! En pleine salle de classe ${ }^{65}$ !»

Les dissidents évoquent ainsi le fait que les autres auraient «vendu la lutte»: ce registre d'énonciation de la contestation fait écho au langage des fescistes et vient confirmer l'hypothèse d'une reproduction des représentations $\mathrm{du}$ «bon syndicaliste » qui tient tête aux puissants, quitte à utiliser des modes d'action violents, qui ne se laisse pas acheter par le pouvoir et est fidèle aux camarades de lutte ${ }^{66}$. En effet, les dissidents de la Fesci regroupés autour de Guillaume Soro en 2000 s'étaient également retournés contre Charles Blé Goudé, l'accusant d'avoir vendu la lutte en s'étant rallié à Laurent Gbagbo et au FPI.

63. Ibrahima Coulibaly, dit «IB», a été le premier leader de la rébellion ivoirienne, mais a été écarté en juin 2004, laissant Guillaume Soro emporter le leadership.

64. Les soulèvements qui eurent lieu en zone rebelle suite aux accords politiques de Ouagadougou et à l'accession de Guillaume Soro au poste de Premier ministre sont ici révélateurs de cette tension. Le remplacement de Koné Zakaria par Wattao dans la zone de commandement de Séguéla atteste de la volonté du clan Soro de faire taire les oppositions, Koné Zakaria ayant exprimé son désaccord publiquement. Voir par exemple l'interview de Koné Zakaria paru le 26 mai 2008 dans le journal Notre Voie. Ce «clan» a par ailleurs été qualifié de «pro-ADO (Alassane Ouattara)», ses partisans voyant la nomination de Soro en tant que Premier ministre comme une trahison vis-à-vis de la cause rebelle qui était uniquement d'installer ADO au pouvoir.

65. Entretien, Bouaké, 1 ${ }^{\mathrm{er}}$ mars 2016.

66. Les logiques de solidarités entre générations fescistes sont extrêmement fortes en Côte d'Ivoire, les membres du syndicat devenant les membres d'une «véritable famille». 


\section{Intégrer la rébellion à l'État}

La mobilisation des enseignants dans le Nord s'acheva par leur intégration à la fonction publique. Le 30 novembre $2007^{67}$, quelques mois après la signature de l'accord de paix de Ouagadougou, Laurent Gbagbo, en visite officielle à Korhogo, annonça le recrutement de l'ensemble des enseignants volontaires du Nord ${ }^{68}$. En avril 2010, ils furent 2665 à être effectivement affectés à un poste d'enseignant sans avoir passé le concours ${ }^{69}$, ainsi que 2429 enseignants volontaires du Sud. Mes enquêtés n'ont cependant jamais évoqué le fait de s'être mobilisés pour l'intégration des volontaires du Sud et me disaient que si le nombre final d'intégrés était de 5094 et non de 2665, c'était du fait de l'inscription de proches des volontaires du Nord, mais surtout d'un ajout auquel le ministère de l'Éducation nationale avait lui-même procédé70.

L'intégration des enseignants volontaires du Nord fit consensus entre les différentes parties en conflit. D'une part, Laurent Gbagbo entretenait des rapports particuliers avec le corps enseignant. Depuis 1976, ils ont de meilleurs salaires que les autres agents de la fonction publique. Or, si en 1991 le premier ministre Alassane Ouattara annonça le retour à l'indexation de leur salaire sur la grille tarifaire classique des fonctionnaires, Laurent Gbagbo y renonça à nouveau en 2003, en pleine guerre. Ceci éclaire la place qu'a pu avoir ce groupe professionnel pour Laurent Gbagbo, qui cherchait à construire une «République des professeurs ${ }^{71}$ », et permet de mieux comprendre sa stratégie de ralliement des enseignants de l'ex-zone rebelle à partir de $2007^{72}$.

D'autre part, l'intégration des enseignants volontaires fut soutenue par les chefs rebelles, politiques comme militaires. Guillaume Soro finança personnellement l'ensemble des visites médicales obligatoires des enseignants de la zone rebelle devant être intégrés. La proximité sociale des chefs rebelles civils avec les étudiants devenus enseignants volontaires joua probablement un rôle déterminant. Souvent très impliqués dans les revendications des jeunes à travers la Fesci dans les années 1990 et début 2000, de nombreux chefs rebelles

67. «Bouaké: 99\% des enseignants volontaires intégrés et payés » [en ligne], @bidj@n.net, 15 janvier 2014. <http://news.abidjan.net/h/484548.html>, consulté le 12 juin 2016.

68. Cette annonce faisait suite à la rentrée scolaire agitée de 2007-2008 où des grèves d'enseignants volontaires avaient été lancées dans la zone rebelle pour réclamer leur intégration.

69. Mémorandum du Mevci, document interne, avril 2010.

70. Entretien, Abidjan, 16 mars 2016.

71. É. Lanoue, «L'école à l'épreuve de la guerre... », art. cité, p. 134 (voir notamment p. 134-140). Éric Lanoue utilise cette expression pour distinguer la politique de Gbagbo de celle de Félix HouphouëtBoigny, pour lequel on parlait de «République des planteurs» (p. 136).

72. Par ailleurs, l'agitation au sein des enseignants volontaires en juin 2007 et la menace d'un blocage du convoi de Gbagbo durant sa visite dans la zone rebelle (symbole de la réconciliation suite aux Accords de Ouagadougou) expliquent également cette décision. 
firent de cette intégration un symbole de l'émancipation des étudiants, de la même manière que la guerre permit la leur. Ce phénomène est propre au Nord, en ce qu'il s'inscrit dans une dynamique lancée par les accords de paix et annonçant l'insertion des anciens agents de l'administration ou de l'armée rebelle à l'État ivoirien dans un contexte de sortie de crise ${ }^{73}$.

L'intégration des enseignants volontaires en Côte d'Ivoire révèle par ailleurs l'existence de deux combats distincts, au Nord et au Sud. Si le Mevci s'est focalisé sur la lutte pour les enseignants de la rébellion, l'insertion parallèle de volontaires au Sud suppose également l'existence d'un mouvement en zone gouvernementale. Cependant, le mouvement du Mevci semble avoir joué un rôle pionnier car le Syndicat des enseignants volontaires de Côte d'Ivoire, représentant les volontaires de la zone gouvernementale, n'est créé qu'après les affectations en avril 2010, et ce dans le but de militer pour la réception des salaires et de la prime de dédommagement non payée. Par ailleurs, le caractère singulier de la lutte syndicale dans le Nord s'illustre par la centralité qu'a prise le Mevci après la guerre, en devenant en 2013 la Fédération des enseignants du secteur éducation formation de Côte d'Ivoire (Fesefci). La Fesefci compte en effet 3000 membres aujourd'hui et est devenue un syndicat national important dans le domaine de l'éducation ${ }^{74}$.

En regardant de plus près les membres actuels de la Fesefci, dont le siège se trouve toujours à Bouaké, on peut voir que l'ensemble des postes clés sont détenus par d'anciens volontaires du Nord, et que le bureau est resté inchangé, incluant ainsi les volontaires ayant intégré le secrétariat général des Forces nouvelles pendant la guerre. Le mouvement syndical ayant existé dans le Nord a joué un rôle central dans le processus de recrutement des volontaires durant le conflit, mais aussi après, comme le montre la généralisation de ce type de pratiques. En effet, les vagues de recrutement de «contractuels ${ }^{75}$ » sont de plus en plus courantes. Un enquêté m'expliquait à ce propos:

«L'État de Côte d'Ivoire a tellement gagné dans cette histoire! Vous savez, madame, l'expérience des enseignants volontaires est devenue inédite en Côte d'Ivoire, je dis pas en Afrique de l'Ouest parce que ça existe dans certains pays. Au Mali, au Sénégal, ils sont pas en crise, mais ils ont ce qu'on appelle les «enseignants vacataires». [...] Mais en Côte d'Ivoire ça n'existait pas en fait. Donc quand y a eu la crise, y a des gens qui ont enseigné

73. Pour les finances, sachant que l'ensemble des régisseurs financiers des régions rebelles étaient des proches de Guillaume Soro et avaient été très actifs à la Fesci, les régisseurs sont devenus cadres dans les Finances publiques ivoiriennes.

74. Voir différents articles de presse, notamment, «Un syndicat plaide pour le reclassement des ex-enseignants volontaires recrutés dans les collèges» [en ligne], @bidj@n.net, 2 septembre 2016, <http://news.abidjan.net/h/598559.html>, consulté le 15 janvier 2018.

75. Le recrutement de contractuels n'est cependant pas nouveau en Côte d'Ivoire, les premiers datant du milieu des années 1980, à travers le recrutement de «licenciés directs ». 
pendant pratiquement huit années scolaires sans percevoir un rond de l'État! Mais ils [les autorités] ont compris que les gens pouvaient enseigner, accepter d'enseigner, pratiquement pour rien! Si simplement on leur promettait l'intégration à la clé. Donc c'est une manière d'avoir du personnel, de la main-d'œuvre bon marché! Donc après les enseignants volontaires, ils ont fait le remake avec les enseignants contractuels ${ }^{76}$.»

La description de ce nouveau mode de recrutement contractuel fait écho à la manière dont les volontaires de la zone rebelle ont été embauchés puis recrutés dans la fonction publique. Outre la reproduction de certaines pratiques d'assimilation de leaders syndicaux, l'histoire des enseignants volontaires durant la guerre montre que la crise ivoirienne semble également avoir créé un précédent dans la gestion des enseignants en Côte d'Ivoire, constat qui est aussi à replacer dans le contexte des discours néolibéraux émis par les organisations internationales, et suivis par le gouvernement d'Alassane Ouattara, visant à réduire les dépenses de l'État par une baisse du nombre de recrutements ou par une précarisation des fonctionnaires, avec par exemple un usage croissant de contrats limités dans le temps.

Si la rébellion ivoirienne n'a rien de proprement révolutionnaire du point de vue de son projet de société, l'intégration des enseignants volontaires à l'État laisse penser que la guerre est un moment de reproduction et peut-être de renforcement des pratiques historiques de l'État ivoirien. Le cas de l'école illustre des dynamiques présentes dans le reste de l'administration de la zone rebelle, dessinant ainsi l'hypothèse d'un processus d'assimilation réciproque des élites où l'intégration à la fonction publique joue un rôle central77.

Si la captation historique des différents segments élitaires mène à la stabilité des régimes, l'inverse n'est pas toujours vrai: l'analyse du secteur scolaire en zone rebelle et des trajectoires de ses acteurs en période post-crise permet en effet d'avancer l'idée que les périodes d'instabilité peuvent donner lieu à des processus d'assimilation encore plus féconds. Le travail d'unification de la part des groupes dominants se retrouve dans les moments de transition et de «réconciliation», et autorise alors un potentiel renforcement du mode de gouvernement en place. Si Laurent Gbagbo a perdu le pouvoir, le fonctionnement de l'État et les modes d'exercice du pouvoir ont été consolidés.

Plus encore, le processus d'assimilation semble avoir visé le segment scolaire de la «nouvelle élite» issue des FN, du fait de la relation de patronage créée entre ex-fescistes rebelles et étudiants. L'intégration des Nordistes dans l'ensemble des administrations étatiques et des postes politiques suggère la 
formation d'une élite, notamment représentée par les patrons syndicaux issus de la Fesci des années 1990, début 200078, et par les jeunes socialisés dans le syndicalisme et la lutte étudiante durant la période de guerre. De ce point de vue, le soutien des ex-fescistes rebelles aux étudiants de la zone CNO et à leur intégration dans l'État contribue à renforcer le pouvoir des leaders syndicaux de la rébellion dans la période post-crise. Cette montée en puissance des acteurs socialisés dans la lutte est intimement liée au mode de gouvernement mis en place dans la zone rebelle: par l'omniprésence des ex-fescistes dans les réseaux politiques rebelles, les étudiants se sont fait une place dans l'État et les syndicalistes étudiants sont devenus un des groupes centraux du jeu politique ivoirien après la crise

Camille Popineau

Université Paris 1

Centre européen de sociologie et de science politique (CESSP)

\section{Abstract \\ Taking up the Chalk. The Mobilization of Rebel Teachers in Northern Côte d'Ivoire (2002-2011)}

This article analyzes trajectories of volunteer teachers in the rebel zone of Côte $d^{\prime}$ lvoire between 2002 and 2011. It shows that patronage networks within the rebellion, the structuration of the school sector, as well as the presence of an imaginary of student unionism as a vector of social upward mobility allowed the mobilization of a group of people with high educational resources. Through their involvement in teachers' unions, they were able to integrate the rebel administration and, later, the State. War reinforced activism and violence as resources to take position in the Ivoirian State. The trajectories of volunteer teachers are an indication that, in times of war, classical ways of social mobility as well as ways of exercising power can be reproduced, thereby questioning the effects of the Ivoirian crisis on power relations within the State.

78. À ce titre, le rôle de président de l'Assemblée nationale, tenu par Guillaume Soro encore aujourd'hui, est symbolique. 